\title{
Quorum Sensing Inhibitors against Chromobacterium violaceum CV026 Derived from an Actinomycete Metabolite Library
}

\author{
Toshiko Ohta, Atsushi Fukumoto, Yohei Iizaka, Fumio Kato, Yasumasa Koyama, and \\ Yojiro Anzai* \\ Faculty of Pharmaceutical Sciences, Toho University; 2-2-1 Miyama, Funabashi, Chiba 274-8510, Japan. \\ Received July 9, 2019; accepted October 1, 2019
}

Quorum sensing (QS) is a microbial signaling system that regulates the expression of many virulence genes. Herein, we studied five compounds-No. 1: $(E)$-2-methyl-3- (4-nitro-phenyl)-acrylaldehyde; No. 29-2: pimprinine [5-(1H-indol-3-yl)-2-methyloxazole]; No. 48: $(2 E, 4 E)-2$-methyl-5-phenyl-2,4-pentadienoic acid; No. 74: (3E,5E)-5-methyl-6-(4-nitrophenyl)-hexa-3,5-dien-2-ol; and No. 130: methyphenazine-1-carboxylate-derived from an actinomycete metabolite library. These compounds were confirmed to be QS inhibitors that reduced violacein production in Chromobacterium violaceum CV026. Additionally, compounds No. 1, No. 74, and No. 130 significantly reduced fluorescent pigment production in Pseudomonas aeruginosa ATCC 27853.

Key words quorum sensing inhibitor; Pseudomonas aeruginosa; actinomycete metabolite library

\section{INTRODUCTION}

Quorum sensing (QS) is a signal transduction system in bacteria that is mediated by substances excreted from bacterial cells into the environment. ${ }^{1)}$ This system regulates the expression of many virulence genes, including those encoding proteins for biofilm formation, ${ }^{2)}$ swarming motility, ${ }^{3)}$ antibiotic biosynthesis, ${ }^{4,5)}$ and virulence factor production. ${ }^{6,7)} \mathrm{A}$ novel strategy to control microbial infections involves the use of agents that inhibit microbial virulence without inhibiting growth, because less selection pressure would generate less microbial resistance. ${ }^{8,9)}$

The disruption of the QS system in pathogenic Burkholderia cepacia and Burkholderia pseudomallei results in reduced pathogenicity in rat and mouse models of infection. ${ }^{10,11)}$ At concentrations below the minimum inhibitory concentration (MIC), erythromycin inhibits biofilm formation in Pseudomonas aeruginosa. ${ }^{12)}$ LED209 is a non-toxic compound that does not inhibit pathogen growth but markedly inhibits the virulence of several pathogens in vitro and in vivo. ${ }^{13)}$ Therefore, compounds that inhibit QS have great potential for use in the treatment of bacterial infectious diseases.

Based on these novel "anti-infective drugs," we previously established a screening system using Chromobacterium violaceum $\mathrm{CV}^{2} 6^{14)}$ to identify QS inhibitors produced by actinomycetes. Piericidin E, a novel compound, was recently discovered in Streptomyces sp. TOHO-Y209 culture broth. $\left.{ }^{15}\right)$ Furthermore, Streptomyces sp. TOHO-M025 was found to produce novel compounds maniwamycins $\mathrm{C}-\mathrm{F}$ that inhibited QS in C. violaceum CV026. ${ }^{16)}$

In this study, the QS inhibition activity of 322 samples from our actinomycete metabolite library was evaluated using a screening system with $C$. violaceum CV026. In addition, these active compounds were tested for their effect on fluorescent pigment production by $P$. aeruginosa.

\section{MATERIALS AND METHODS}

Bacterial Strains, Media, and Culture $C$. violaceum $\mathrm{CV}^{2} 6^{14)}$ was cultured in Luria Broth [LB; 1.0\% Bacto tryptone (Becton, Dickinson and Company, Sparks, MD, U.S.A.), $0.5 \%$ Bacto yeast extract (Becton, Dickinson and Company), and $0.5 \% \mathrm{NaCl}$ (Wako Pure Chemical Corporation, Osaka, Japan), $\mathrm{pH} 7.2$ ] at $27^{\circ} \mathrm{C}$. P. aeruginosa ATC C $27853,{ }^{17)}$ a quality control strain for dilution antimicrobial susceptibility tests, was cultured overnight in Nutrient Broth (NB; Eiken Chemical Co., Ltd., Tochigi, Japan) at $37^{\circ} \mathrm{C}$.

Screening Samples In total, 322 samples from our actinomycete metabolite library, consisting of compounds obtained from actinomycete cultures and synthesized as partial structures of actinomycete metabolites, were used for screening. These compounds were stored by Professors Yasumasa Koyama and Fumio Kato, who studied metabolites (including antibiotics) produced by actinomycetes 40 to 50 years ago. The structures of the active samples were confirmed by ${ }^{1} \mathrm{H}-\mathrm{NMR}$ (500 MHz) spectroscopy (JNM-ECA500, JEOL Ltd., Tokyo, Japan) and mass spectrometry (LCMS-8040, Shimadzu Co., Kyoto, Japan).

QS Inhibition Assay The microtiter plate assay was performed for QS inhibition screening, according to our previous study. ${ }^{16)}$ In the checkerboard assay, 0.0625, 0.125, 0.25 , or $0.5 \mathrm{mg} / \mathrm{mL}$ of the test compound and $0.008,0.04$, or $0.2 \mathrm{mg} / \mathrm{mL}$ of $N$-hexanoyl-L-homoserine lactone $(N$-HHL; Santa Cruz Biotech. Co., Santa Cruz, CA, U.S.A.) were added to each well.

Effect of Compounds on Fluorescent Pigment Production by $\boldsymbol{P}$. aeruginosa In each well of a 96-well cell culture plate (CELLSTAR ${ }^{\circledR} 96$ well plates No. 655180, Greiner BioOne GmbH, Frickerhansen, Germany), $200 \mu \mathrm{L}$ of NB containing $0.5 \mu \mathrm{L}$ of $P$. aeruginosa ATCC 27853 culture broth and $0.5 \mathrm{mg} / \mathrm{mL}$ of the test compound were added. The microtiter plate was incubated at $37^{\circ} \mathrm{C}$ for $24 \mathrm{~h}$ with shaking. Equal volumes of the culture broth $(100 \mu \mathrm{L})$ and dimethyl sulfoxide (DMSO) $(100 \mu \mathrm{L})$ were added to each well of a 96 -well black 
plate Nunc $^{\mathrm{TM}}$ F96 MicroWell $^{\mathrm{TM}}$ Black Polystyrene Plate No. 137101, Thermo Fisher Scientific, Roskilde, Denmark), and fluorescence intensity at an absorption wavelength of $470 \mathrm{~nm}$
No. 1<smiles>CC(=CC=Cc1ccccc1)C(=O)O</smiles>

No. 48<smiles>COC(=O)c1cccc2nc3ccccc3nc12</smiles>

No. 130<smiles>CC(C=O)=Cc1ccc([N+](=O)[O-])cc1</smiles><smiles>Cc1ncc(-c2c[nH]c3ccccc23)o1</smiles>

No. $29-2$<smiles>CC(/C=C/C(C)O)=C\c1ccc([N+](=O)[O-])cc1</smiles>

No. 74<smiles>CCCCCC(=O)NC1CCOC1=O</smiles>

$\mathrm{N}-\mathrm{HHL}$
Fig. 1. Structures of Compound No. 1, No. 29-2, No. 48, No. 74, No. 130 , and $N$-Hexanoyl-L-homoserine Lactone ( $N$-HHL)

No. 1

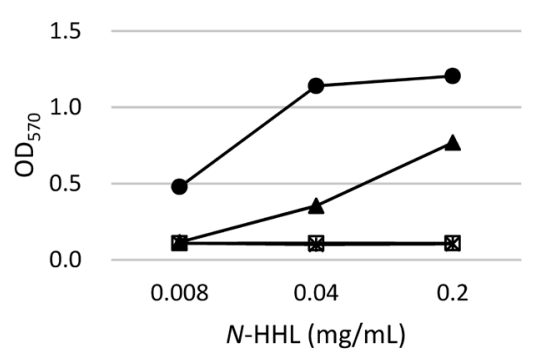

No. 48

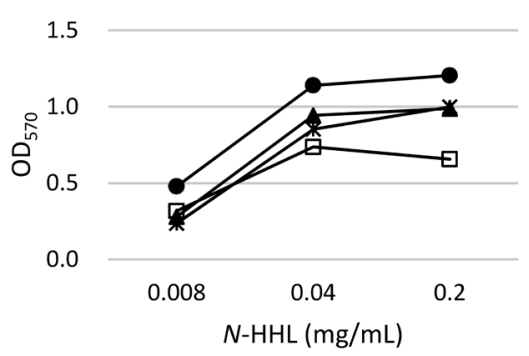

No. 130

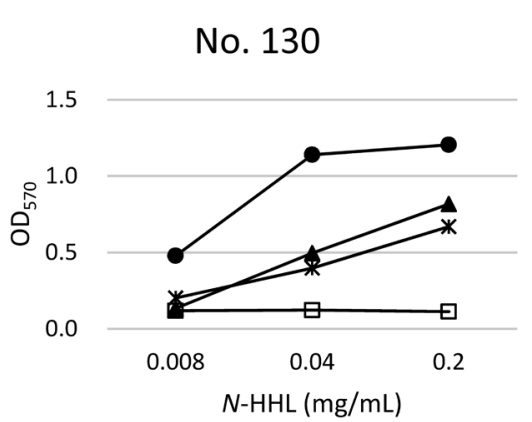

and excitation wavelength of $365 \mathrm{~nm}$ was measured using a multimode plate reader EnSpire2300 (PerkinElmer, Inc., MA, U.S.A.).

Antibacterial Activity and Bacterial Growth Rate The antibacterial activities of the compounds for $C$. violaceum CV026 and $P$. aeruginosa ATCC 27853 were evaluated by determining MICs using the microbroth dilution method with Mueller-Hinton medium (Becton, Dickinson and Company). The test compounds were serially diluted and added to the growth medium in 96-well plates. Each well was inoculated with the tested bacterial strains and incubated at 27 or $37^{\circ} \mathrm{C}$ for $20-24 \mathrm{~h}$. Bacterial growth was evaluated by $\mathrm{OD}_{600}$ measurement. The growth rates of both strains were also evaluated under pigment production culture conditions through $\mathrm{OD}_{600}$ measurement in 96-well plates. C. violaceum CV026 was cultured in LB soft agar \pm test compound $(0.5 \mathrm{mg} / \mathrm{mL})$ in the absence of $N$-HHL.

\section{RESULTS}

The QS inhibitory activity was determined using C. violaceum CV026 and 322 samples from the actinomycete metabolite library. At a concentration of $1 \mathrm{mg} / \mathrm{mL}$, five compounds

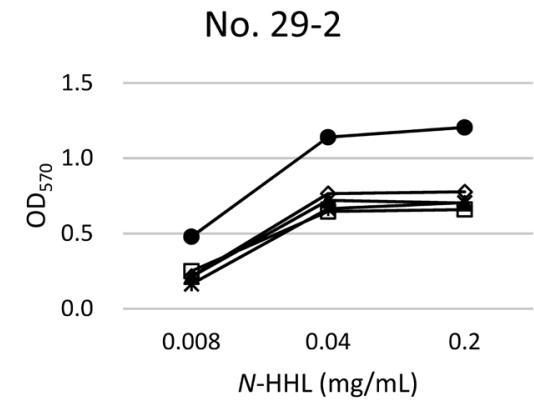

No. 74

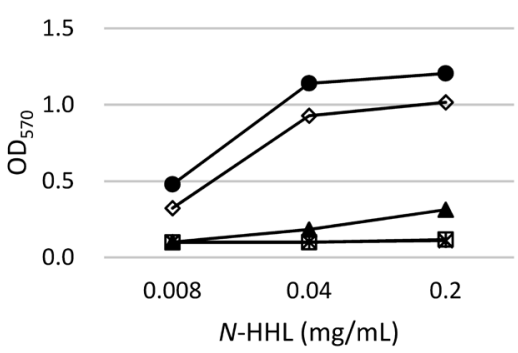

Fig. 2. Results of the Checkerboard Assay between QS Inducer $N$-HHL and the Compounds Selected for QS Inhibition Screening

Violacein production is plotted as the concentration of $N$-HHL for each QS inhibitor concentration (circle: $0 \mathrm{mg} / \mathrm{mL}$, rhombus: $0.0625 \mathrm{mg} / \mathrm{mL}$, triangle: $0.125 \mathrm{mg} / \mathrm{mL}$, asterisk: $0.25 \mathrm{mg} / \mathrm{mL}$, and square: $0.5 \mathrm{mg} / \mathrm{mL}$ ). 


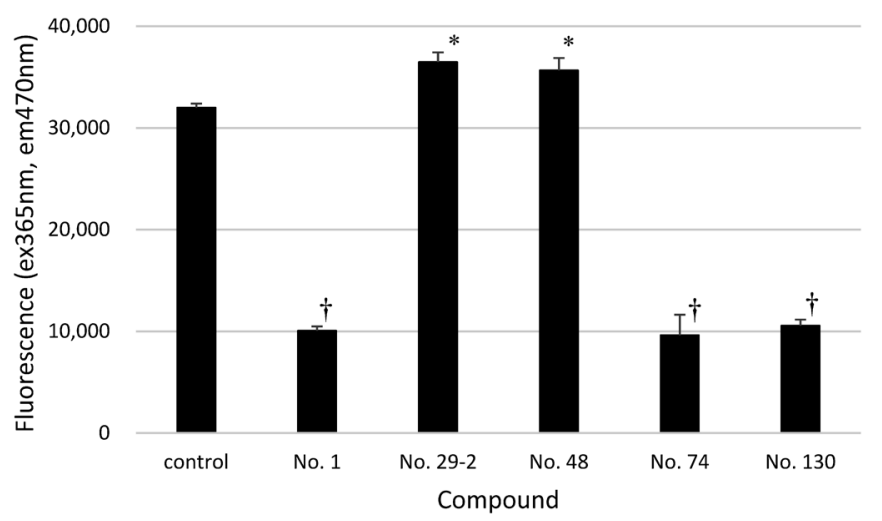

Fig. 3. Effect of Test Compounds on Fluorescent Pigment Production in $P$. aeruginosa ATCC 27853

Each compound $(0.5 \mathrm{mg} / \mathrm{mL})$ was added to $200 \mu \mathrm{L}$ NB with $0.5 \mu \mathrm{L}$ pre-culture broth. After incubation at $37^{\circ} \mathrm{C}$ for $24 \mathrm{~h}$, the excitation spectra for the fluorescent pigment in culture broth was measured at an emission wavelength of $470 \mathrm{~nm}$ and excitation wavelength of $365 \mathrm{~nm}$. Error bars represent the standard error of the mean for three biological replicates. ${ }^{*}$ indicates a significant increase compared to the control (no compound) at $p<0.05$. $\dagger$ indicates a significant decrease compared to the control at $p<0.05$.

reduced violacein production in $C$. violaceum to approximately half or less of its original value. As the structural formulae of other compounds were indeterminable in some samples from the library, the structures of these five compounds were confirmed (data not shown). These five compounds were identified as: No. 1, (E)-2-methyl-3-(4-nitro-phenyl)-acrylaldehyde; No. 29-2, pimprinine [5-(1H-indol-3-yl)-2-methyloxazole]; No. 48, $(2 E, 4 E)-2$-methyl-5-phenyl-2,4-pentadienoic acid; No. 74, (3E,5E)-5-methyl-6-(4-nitrophenyl)-hexa-3,5-dien-2-ol; and No. 130, methyphenazine-1-carboxylate (Fig. 1).

A checkerboard assay was performed to determine if these compounds competed with $N$-HHL, which was used as an inducer of QS (Fig. 2). Violacein production was completely suppressed by No. 1 and No. 74 at $0.25 \mathrm{mg} / \mathrm{mL}$ and by No. 130 at $0.5 \mathrm{mg} / \mathrm{mL}$. A concentration-dependent competitive inhibition of $N$-HHL was observed below these concentrations for No. 1, No. 74, and No. 130. In contrast, No. 29-2 and No. 48 showed a concentration-dependent partial inhibition of $\mathrm{N}$-HHL; moreover, violacein production was not completely suppressed, even at $0.5 \mathrm{mg} / \mathrm{mL}$.

The effects of these five compounds on fluorescent pigment production by $P$. aeruginosa were examined (Fig. 3). At $0.5 \mathrm{mg} / \mathrm{mL}$, No. 1 , No. 74 , and No. 130 decreased fluorescent pigment production by 69,70 , and $67 \%$, respectively. In contrast, No. 29-2 and No. 48 increased fluorescent pigment production by 14 and $11 \%$, respectively, relative to the control.

The antibacterial activities of these compounds against $C$. violaceum and $P$. aeruginosa were tested based on the Clinical and Laboratory Standards Institute (CLSI) methods using Mueller-Hinton medium. No. 1 showed no antibacterial activity against any strain at concentrations from 1 to $512 \mu \mathrm{g} / \mathrm{mL}$. However, $P$. aeruginosa culture turbidity decreased by $42 \%$ at $1024 \mu \mathrm{g} / \mathrm{mL}$ No. 1 . The other compounds showed no antibacterial activity, even at $1024 \mu \mathrm{g} / \mathrm{mL}$; moreover, none showed growth-inhibitory effects against $C$. violaceum.

The 24-h growth curve for each of these bacteria was determined under pigment production conditions to confirm the effects of these compounds (Fig. 4). The C. violaceum CV026 growth curves with No. 1 and No. 29-2 were similar to that of the control from 0 to $24 \mathrm{~h}$, whereas No. 74 slightly suppressed
A

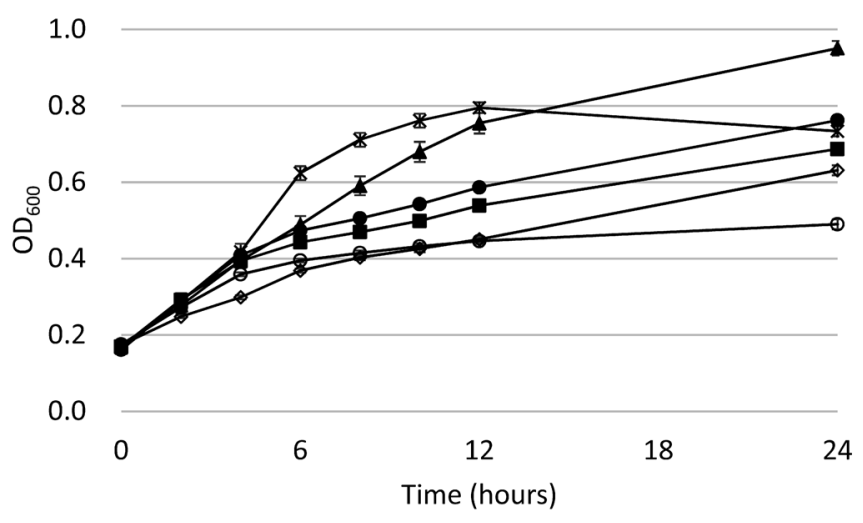

B

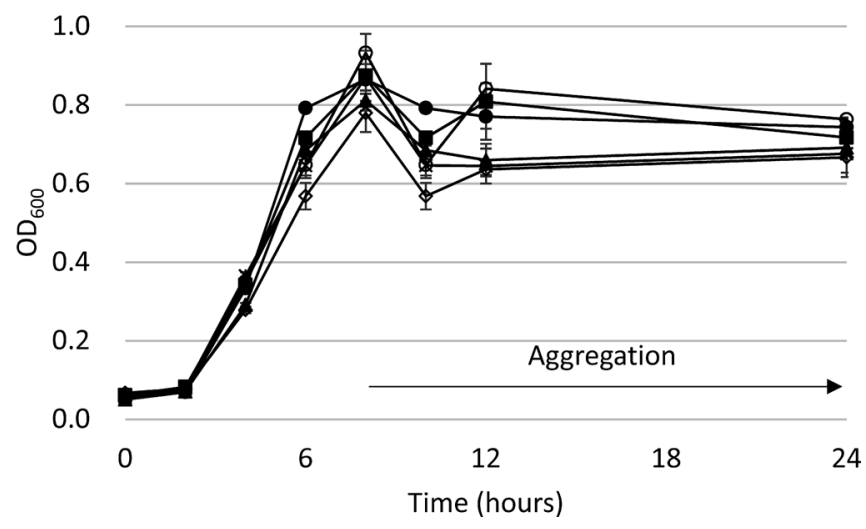

Fig. 4. Growth Curves of C. violaceum CV026 (A) and P. aeruginosa ATCC 27853 (B) with $0.5 \mathrm{mg} / \mathrm{mL}$ of Each Compound (Solid Circle: No Compound, Rhombus: No. 1, Square: No. 29-2, Triangle: No. 48, Open Circle: No. 74, and Asterisk: No. 130)

C. violaceum $\mathrm{CV} 026$ was cultured in LB soft agar \pm compound without $N$-HHL at $27^{\circ} \mathrm{C}$. P. aeruginosa ATCC 27853 was cultured in $\mathrm{NB} \pm$ compound at $37^{\circ} \mathrm{C}$ with shaking. After $8 \mathrm{~h}, P$. aeruginosa ATCC 27853 cells began to aggregate in each well. Error bars represent the standard error of the mean for three biological replicates.

C. violaceum CV026 growth after 12 h. C. violaceum CV026 treated with No. 48 and No. 130 grew better than the control during the intermediate-to-late growth stage (6-12h). After $24 \mathrm{~h}$, the $\mathrm{OD}_{600}$ of the $C$. violaceum CV026 culture treated with No. 48 reached 0.95 , whereas that treated with No. 130 reached 0.73 , similar to that of the control. In contrast, the growth rate of $P$. aeruginosa ATCC 27853 was not affected at all by these compounds. During the initial growth stage $(0-8 \mathrm{~h})$, the $\mathrm{OD}_{600}$ of the cultures treated with the test compounds as well as the control was increased. However, after $8 \mathrm{~h}$, the cells began to aggregate and the $\mathrm{OD}_{600}$ varied. This strain grew planktonically up to $8 \mathrm{~h}$ and aggregated thereafter. The $\mathrm{OD}_{600}$ of the cultures treated with the test compounds was similar to that of the control after $24 \mathrm{~h}$.

\section{DISCUSSION}

None of the five compounds that reduced violacein production in $C$. violaceum have been previously reported as QS inhibitors. No. 1, (E)-2-methyl-3-(4-nitro-phenyl)-acrylaldehyde, was previously reported as a metabolite produced by Streptomyces luteoreticuli. Moreover, unsaturated ketone derivatives of sphenyl-acrolein have been shown to exhibit antimicrobial 
activity. ${ }^{18)}$ An unsaturated ketone derivative of No. 1, synthesized by Gasha et al., inhibited the growth of Trichophyton mentagrophytes. ${ }^{19)}$ No. 29-2, pimprinine [5-(1H-indol-3-yl)2-methyloxazole] - a metabolite produced by Streptomyces pimprina - ${ }^{20)}$ was reported to exhibit anti-tuberculosis activity. $^{21)}$ No. 48, (2E,4E)-2-methyl-5-phenyl-2,4-pentadienoic acid, was synthesized by Koyama et al. and used for structure determination of luteoreticulin produced by $S$. luteoreticuli. ${ }^{18)}$ No. 74, (3E,5E)-5-methyl-6-(4-nitrophenyl)-hexa-3,5-dien-2-ol, was synthesized as a derivative of aureothin, which was isolated as a by-product of the antibiotic aureothricin from Streptomyces thioluteus. ${ }^{22)}$ No. 130, methyphenazine-1-carboxylate, was isolated as a metabolite from S. luteoreticuli in 1971. ${ }^{23)}$ Recently, No. 130 was reported as a metabolite produced by Pseudomonas chlororaphis and strains of the genus Kitasatospora; moreover, the antifungal and anti-Gram-positive-bacterial activities of No. 130 were demonstrated. ${ }^{24,25)}$

As No. 1, No. 74, and No. 130 act competitively with $N$-HHL, an inducer of QS, in C. violaceum, these compounds can inhibit the activity of the transcription factor CviR, which is induced by $N$-HHL in $C$. violaceum CV026. Interestingly, the structures of No. 1 and No. 74 are very similar, and the sub-structures of both compounds consist of 1-nitro-4-(1(1E)-propenyl)-benzene. Moreover, in our study, these three compounds significantly reduced fluorescent pigment production in $P$. aeruginosa. On the contrary, all five compounds showed no effect on the production of the green pigment pyocyanin, which is positively regulated by the RhlR-RhlI QS system. ${ }^{26)} P$. aeruginosa ATCC 27853 did not produce pyocyanin for up to $24 \mathrm{~h}$; however, pyocyanin was detected after $2 \mathrm{~d}$. There was no difference in pyocyanin production on the next day, when fluorescent pigment production was observed (data not shown). The QS response of $P$. aeruginosa primarily depends on two transcriptional regulators of the LuxR family, LasR and RhlR. ${ }^{27)}$ Moreover, the active site of LasR is structurally similar to that of CviR. ${ }^{28)}$ Therefore, it is presumed that No. 1, No. 74, and No. 130 bind to the LasR protein of $P$. aeruginosa. The production of a fluorescent pigment pyoverdine by $P$. aeruginosa is reduced in the lasI/las $R$ mutant ${ }^{29)}$ and enhanced by the cell-signaling protein PqsA. ${ }^{30}$ The transcription of $p q s \mathrm{~A}$ is induced by the third transcriptional regulator PqsR, which is positively regulated by the LasR/N-3-oxododecanoyl-L-homoserine complex. ${ }^{31)}$ However, as the growth curves of $C$. violaceum CV026 treated with No. 74 and No. 130 were slightly different from that of the control, the QS inhibition system of these active compounds including No. 1 should be further investigated. Conversely, No. 29-2 and No. 48 slightly increased fluorescent pigment production in $P$. aeruginosa. As No. 29-2 and No. 48 only partially competed with $N$-HHL in $C$. violaceum, CviR function might have been partially inhibited. The fluorescence intensity (excitation $365 \mathrm{~nm}$, absorption $470 \mathrm{~nm}$ ) of the compounds No. 29-2 and No. 48 was not detected (data not shown). However, as the increase in fluorescent pigment production was very less, this phenomenon may have been influenced by growth variations caused by cell aggregation during culture.

Several new compounds, such as piericidin E and maniwamycins $\mathrm{C}-\mathrm{F}$, have been discovered in actinomycete culture broths and were used in our QS inhibitor screening study. ${ }^{15,16)}$ In this study, five compounds from a library of actinomycete metabolites and their derivatives were identified as QS inhibi- tors against $C$. violaceum. Additionally, they displayed positive or negative effects on fluorescent pigment production by $P$. aeruginosa. Thus, these QS inhibitors can be potential lead compounds for the creation of more potent and specific QS inhibitors for further studies of QS systems.

Acknowledgments We thank Dr. Tsukasa Ikeda at Utsunomiya University for kindly providing C. violaceum CV026. This work was supported by Private University Research Branding Project from the Ministry of Education, Culture, Sports, Science and Technology (MEXT) of Japan.

Conflict of Interest The authors declare no conflict of interest.

\section{REFERENCES}

1) Bassler BL, Losick R. Bacterially speaking. Cell, 125, 237-246 (2006).

2) McLean RJ, Whiteley M, Stickler DJ, Fuqua WC. Evidence of autoinducer activity in naturally occurring biofilms. FEMS Microbiol. Lett., 154, 259-263 (1997).

3) Eberl L, Winson MK, Sternberg C, Stewart GS, Christiansen G, Chhabra SR, Bycroft B, Williams P, Molin S, Givskov M. Involvement of $N$-acyl-L-hormoserine lactone autoinducers in controlling the multicellular behaviour of Serratia liquefaciens. Mol. Microbiol., 20, 127-136 (1996).

4) Slater H, Crow M, Everson L, Salmond GP. Phosphate availability regulates biosynthesis of two antibiotics, prodigiosin and carbapenem, in Serratia via both quorum-sensing-dependent and -independent pathways. Mol. Microbiol., 47, 303-320 (2003).

5) Fineran PC, Slater H, Everson L, Hughes K, Salmond GP. Biosynthesis of tripyrrole and beta-lactam secondary metabolites in Serratia: integration of quorum sensing with multiple new regulatory components in the control of prodigiosin and carbapenem antibiotic production. Mol. Microbiol., 56, 1495-1517 (2005).

6) Falcão JP, Sharp F, Sperandio V. Cell-to-cell signaling in intestinal pathogens. Curr. Issues Intest. Microbiol., 5, 9-17 (2004).

7) Antunes LC, Ferreira RB, Buckner MM, Finlay BB. Quorum sensing in bacterial virulence. Microbiology, 156, 2271-2282 (2010).

8) Finch RG, Pritchard DI, Bycroft BW, Williams P, Stewart GS. Quorum sensing: a novel target for anti-infective therapy. J. Antimicrob. Chemother., 42, 569-571 (1998).

9) Cegelski L, Marshall GR, Eldridge GR, Hultgren SJ. The biology and future prospects of antivirulence therapies. Nat. Rev. Microbiol., 6, 17-27 (2008).

10) Baldwin A, Sokol PA, Parkhill J, Mahenthiralingam E. The Burkholderia cepacia epidemic strain marker is part of a novel genomic island encoding both virulence and metabolism-associated genes in Burkholderia cenocepacia. Infect. Immun., 72, 1537-1547 (2004).

11) Valade E, Thibault FM, Gauthier YP, Palencia M, Popoff MY, Vidal DR. The PmlI-PmlR quorum-sensing system in Burkholderia pseudomallei plays a key role in virulence and modulates production of the MprA protease. J. Bacteriol., 186, 2288-2294 (2004).

12) Nagata T, Mukae H, Kadota J, Hayashi T, Fujii T, Kuroki M, Shirai R, Yanagihara K, Tomono K, Koji T, Kohno S. Effect of erythromycin on chronic respiratory infection caused by Pseudomonas aeruginosa with biofilm formation in an experimental murine model. Antimicrob. Agents Chemother., 48, 2251-2259 (2004).

13) Rasko DA, Moreira CG, Li R, Reading NC, Ritchie JM, Waldor MK, Williams N, Taussig R, Wei S, Roth M, Hughes DT, Huntley JF, Fina MW, Falck JR, Sperandio V, Li de R, Reading NC, Ritchie JM, Waldor MK, Williams N, Taussig R, Wei S, Roth M, Hughes DT, Huntley JF, Fina MW, Falck JR, Sperandio V. Targeting QseC signaling and virulence for antibiotic development. Science, 321, 
1078-1080 (2008).

14) McClean KH, Winson MK, Fish L, Taylor A, Chhabra SR, Camara M, Daykin M, Lamb JH, Swift S, Bycroft BW, Stewart GS, Williams P. Quorum sensing and Chromobacterium violaceum: exploitation of violacein production and inhibition for the detection of $N$-acylhomoserine lactones. Microbiology, 143, 3703-3711 (1997).

15) Ooka K, Fukumoto A, Yamanaka T, Shimada K, Ishihara R, Anzai Y, Kato F. Novel Quorum-sensing inhibitors against Chromobacterium violaceum $\mathrm{CV} 026$, from Streptomyces $\mathrm{sp}$. TOHO-Y209 and TOHO-O348. Open J. Med. Chemistry, 3, 93-99 (2013).

16) Fukumoto A, Murakami C, Anzai Y, Kato F. Maniwamycins: new quorum-sensing inhibitors against Chromobacterium violaceum CV026 were isolated from Streptomyces sp. TOHO-M025. J. Antibiot., 69, 395-399 (2016).

17) "Pseudomonas aeruginosa (Schroeter) Migula (ATCC $\left.{ }^{\circledR} 27853^{\mathrm{TM}}\right)$.": 〈https://www.atcc.org/products/all/27853.aspx〉, accessed 29 August, 2019.

18) Koyama Y, Kyomura N, Onishi J, Yamaguchi S. On the metabolites of Streptomyces luteoreticuli KATOH et ARAI. III. The structure of luteoreticulum. (2). Yakugaku Zasshi, 95, 1210-1217 (1975).

19) Gasha M, Tsuji A, Sakurai Y, Kurumi M, Endo T, Sato S, Yamaguchi K. Antimicrobial activity of 5-oxothiazolidine-2-thione and rhodanine derivatives. Yakugaku Zasshi, 92, 490-497 (1972).

20) Koyama Y, Yokose K, Dolby LJ. Isolation, characterization and synthesis of pimprinine, pimprinethine and pimprinaphine, metabolites of Streptoverticillium olivoreticuli. Agric. Biol. Chem., 45, 1285-1287 (1981).

21) Intaraudom $C$, Rachtawee $P$, Suvannakad R, Pittayakhajonwut $P$. Antimalarial and antituberculosis substances from Streptomyces $\mathrm{sp}$. BCC26924. Tetrahedron, 67, 7593-7597 (2011).

22) Hirata $Y$, Nakata $H$, Yamada $K$, Okuhara $K$, Naito T. The structure of aureothin, a nitro compound obtained from Streptomyces thioluteus. Tetrahedron, 14, 252-274 (1961).
23) Yamagishi S, Koyama Y, Fukakusa Y, Kyomura N, Ohishi J. On the metabolites of Streptomyces luteoreticuli KATOH et ARAI. I. Isolation of the metabolites. Yakugaku Zasshi, 91, 351-357 (1971).

24) $\overline{\mathrm{Wu}} \mathrm{C}$, van Wezel GP, Hae Choi Y. Identification of novel endophenaside antibiotics produced by Kitasatospora sp. MBT66. J. Antibiot., 68, 445-452 (2015).

25) Puopolo G, Masi M, Raio A, Andolfi A, Zoina A, Cimmino A, Evidente A. Insights on the susceptibility of plant pathogenic fungi to phenazine-1-carboxylic acid and its chemical derivatives. Nat. Prod. Res., 27, 956-966 (2013).

26) Brint JM, Ohman DE. Synthesis of multiple exoproducts in Pseudomonas aeruginosa is under the control of RhlR-RhlI, another set of regulators in strain PAO1 with homology to the autoinducerresponsive LuxR-LuxI family. J. Bacteriol., 177, 7155-7163 (1995).

27) Williams P, Cámara M. Quorum sensing and environmental adaptation in Pseudomonas aeruginosa: a tale of regulatory networks and multifunctional signal molecules. Curr. Opin. Microbiol., 12, 182-191 (2009).

28) Gnanendra S, Anusuya S, Natarajan J. Molecular modeling and active site analysis of SdiA homolog, a putative quorum sensor for Salmonella typhimurium pathogenecity reveals specific binding patterns of AHL transcriptional regulators. J. Mol. Model., 18, $4709-4719$ (2012)

29) Stintzi A, Evans K, Meyer JM, Poole K. Quorum-sensing and siderophore biosynthesis in Pseudomonas aeruginosa: lasR/lasI mutants exhibit reduced pyoverdine biosynthesis. FEMS Microbiol. Lett., 166, 341-345 (1998).

30) Kang D, Turner KE, Kirienko NV. PqsA promote Pyoberidine production via Biofilm formation. Pathogens, 7, 3 (2017).

31) Farrow JM III, Pesci EC. Distal and proximal promoters co-regulate pqsR expression in Pseudomonas aeruginosa. Mol. Microbiol., 104, 78-91 (2017). 